\title{
Indirect dark matter searches as a probe of degenerate particle spectra
}

\author{
Masaki Asano ${ }^{\mathrm{a}}$, Torsten Bringmann ${ }^{\mathrm{a}}$, Christoph Weniger ${ }^{\mathrm{b}}$ \\ ${ }^{a}$ II. Institute for Theoretical Physics, University of Hamburg, Luruper Chaussee 149, DE-22761 Hamburg, Germany \\ ${ }^{b}$ Max-Planck-Institut für Physik, Föhringer Ring 6, 80805 Munich, Germany
}

\begin{abstract}
We consider the possibility that the cosmological dark matter consists of particles very close in mass to new colored particles below the $\mathrm{TeV}$ scale. While such a scenario is inherently difficult to directly confirm at colliders, we find that indirect dark matter searches may be a powerful alternative. In particular, we show that in this case dark matter annihilation to $\bar{q} q g$ final states can give rise to significant antiproton (but also gamma-ray) fluxes, and compare the resulting constraints to bounds from direct searches at LEP, the Tevatron and the LHC. For supersymmetric neutralinos degenerate with squarks, e.g., antiprotons can give rise to more stringent constraints for masses below around 45-80 GeV.
\end{abstract}

\section{Introduction}

Collider experiments have pushed the scale for possible new physics beyond the standard model (BSM) to ever higher energies in recent years. The CERN LHC, in particular, is now running at a center of mass energy of $7 \mathrm{TeV}$ and the non-observation of any clear BSM signal, so far, has allowed to place impressive limits [1, 2]; after an integrated luminosity of $1.04 \mathrm{fb}^{-1}$, scalar quarks (squarks) in supersymmetric extensions of the standard model, e.g., have already been excluded for masses below $\sim \sim 900 \mathrm{GeV}$ [3, 4]. One has to keep in mind, however, that these results rest on the assumption that the new colored states quickly decay into the lightest neutralino, which is assumed to be massless for the sake of the analysis, and emit high-energy QCD jets in this process. These limits thus do not apply if the only accessible lighter states are very close in mass $[5,6,7,8$, in which case the resulting jets and missing transverse energy would be too soft to pass the signal selection criteria. Scenarios with degenerate particle spectra are thus generically very difficult to probe at hadron colliders; the situation is considerably better for electron-positron colliders like LEP, but even in this case the limits from direct searches for colored states do not apply to highly degenerate spectra [9, 10, 11, 12, 13.

Here, we investigate whether such a situation could be probed by indirect dark matter (DM) searches, assuming that the lightest BSM state is a weakly interacting massive particle (WIMP) that makes up the cosmological DM. The self-annihilation of WIMP pairs could then leave an imprint in the spectrum of cosmic rays (see, e.g., Ref. 14] for a review on particle DM and indirect searches). As we will demonstrate, WIMP annihilation into $\bar{q} q g$ final states,

\footnotetext{
Email addresses: masaki.asano@desy.de (Masaki Asano), torsten.bringmann@desy.de (Torsten Bringmann), weniger@mppmu.mpg.de (Christoph Weniger)
}

a channel so far hardly explored in indirect DM searches, may give rise to the most stringent constraints in degenerate scenarios (mostly through antiproton, but also through gamma-ray production). In fact, this channel may help to fill remaining loop-holes for the existence of new colored states below masses of around $100 \mathrm{GeV}$ that are left from direct collider searches for such particles.

The rest of this Letter is organized as follows: In Section 2 we discuss the importance and main characteristics of DM annihilation into $\bar{q} q g$ final states, focussing on the case of neutralino DM; in Section 3 we present the energy spectra of antiprotons that result from $\bar{q} q g$ hadronization and discuss antiproton propagation through our Galaxy; Section 4 contains the limits on the annihilation crosssection that can be derived from cosmic-ray observations and Section 5 confronts these limits with direct collider searches for charged colored particles. Finally, in Section 6 and 7, respectively, we present a discussion of our results and conclude.

\section{Dark matter annihilation into gluons}

Sizable cosmic-ray fluxes from galactic DM can only be expected from $s$-wave annihilation processes; this is because the $p$-wave rate scales like $v^{2}$ and is heavily suppressed for the typically expected DM velocities of the order of $v \sim 10^{-3}$. However, if the annihilating DM pair is in a $J=0$ state - as is necessarily the case for scalar or Majorana DM particles $\chi$ - the tree-level $s$-wave annihilation rate into fermion pairs $\bar{f} f$ is helicity suppressed and scales like $\sigma v \propto m_{f}^{2} / m_{\chi}^{2}$. In this situation, the existence of an additional vector boson in the final state may lift the helicity suppression and lead to a radiative 'correction' of the order of $(\alpha / \pi) m_{\chi}^{2} / m_{f}^{2}$, which in practice can correspond to an annihilation rate that is enhanced by several orders of magnitude [15, 16]. 

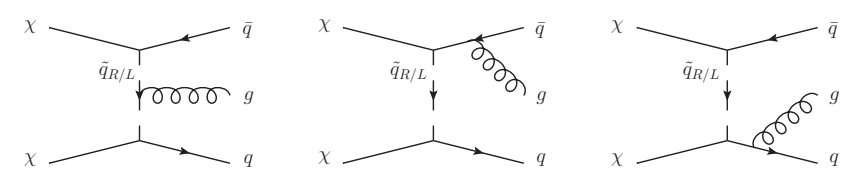

Figure 1: Feynman diagrams contributing to $\chi \chi \rightarrow \bar{q} q g$ in the limit of vanishing quark mass and neutralino velocity.

We will in the following focus on the supersymmetric neutralino, but note that we expect qualitatively very similar results for other DM candidates where the annihilation rate into fermions is helicity suppressed. The annihilation of neutralinos into $\bar{q} q$ two-body final states, in particular, is typically strongly suppressed (at least for $m_{b} \ll m_{\chi}<m_{t}$ or $m_{\chi} \gg m_{t}$ ), so that the dominant contribution to the total annihilation cross section into quarks is given by the process $\chi \chi \rightarrow \bar{q} q g$. In the $m_{q} \rightarrow 0$ limit, only the squarkexchange $t$-channel diagrams shown in Fig. 1 contribute and we find

$$
\begin{aligned}
& (\sigma v)_{v \rightarrow 0}^{\chi \chi \rightarrow \bar{q} q g}=\frac{\alpha_{\mathrm{s}}\left|\tilde{g}_{R}\right|^{4}}{16 \pi^{2} m_{\chi}^{2}} \times\left\{\frac{3+4 \mu_{R}}{1+\mu_{R}}\right. \\
& +\left(1+\mu_{R}\right)\left[\frac{\pi^{2}}{6}-\left(\log \frac{\mu_{R}+1}{2 \mu_{R}}\right)^{2}-2 \operatorname{Li}_{2}\left(\frac{\mu_{R}+1}{2 \mu_{R}}\right)\right] \\
& \left.+\frac{4 \mu_{R}^{2}-3 \mu_{R}-1}{2 \mu_{R}} \log \frac{\mu_{R}-1}{\mu_{R}+1}\right\}+(R \leftrightarrow L)
\end{aligned}
$$

where $\mu_{R, L} \equiv m_{\tilde{q}_{R, L}}^{2} / m_{\chi}^{2}$ and $\tilde{g}_{R} P_{L}\left(\tilde{g}_{L} P_{R}\right)$ is the coupling between neutralino, quark and right-handed (left-handed) squark; $\operatorname{Li}_{2}(z)=\sum_{k=1}^{\infty} z^{k} / k^{2}$ is the dilogarithm. This result is consistent with the differential cross section presented in Ref. [17]; for comparison, it can also be obtained by the corresponding expressions for photon final states $\bar{q} q \gamma$ [18] by simply replacing $Q^{2} \alpha_{\mathrm{em}} \rightarrow(4 / 3) \alpha_{s}$, where $Q$ is the electric charge of $q 1^{1}$

If the neutralino is a pure Bino with $m_{b} \ll m_{\tilde{B}}<m_{t}$, the total annihilation cross section is well approximated by Eq. (1) as long as the mass-splitting $\mu$ remains small. This is thus the minimal neutralino annihilation cross section we can expect; in the exactly degenerate case, $\mu_{R}=\mu_{L}=$ 1 , it is largest and becomes

$$
(\sigma v)_{v \rightarrow 0}^{\tilde{B} \tilde{B} \rightarrow \bar{q} q g}=\frac{2 \alpha_{\mathrm{s}} \alpha_{Y}^{2}}{3 m_{\tilde{B}}^{2}}\left(21-2 \pi^{2}\right)\left(6^{-4}+Q^{4}\right) .
$$

For other neutralino compositions, e.g. if the neutralino is a Wino, one can find higher total cross sections - mainly because annihilation into $W^{+} W^{-}$and $Z Z$ final states becomes effective (but also because the neutralino-(s)quark couplings in Eq. (1) can be larger). However, these scenarios tend to be more constrained than Bino neutralinos, and we will here concentrate on the latter.

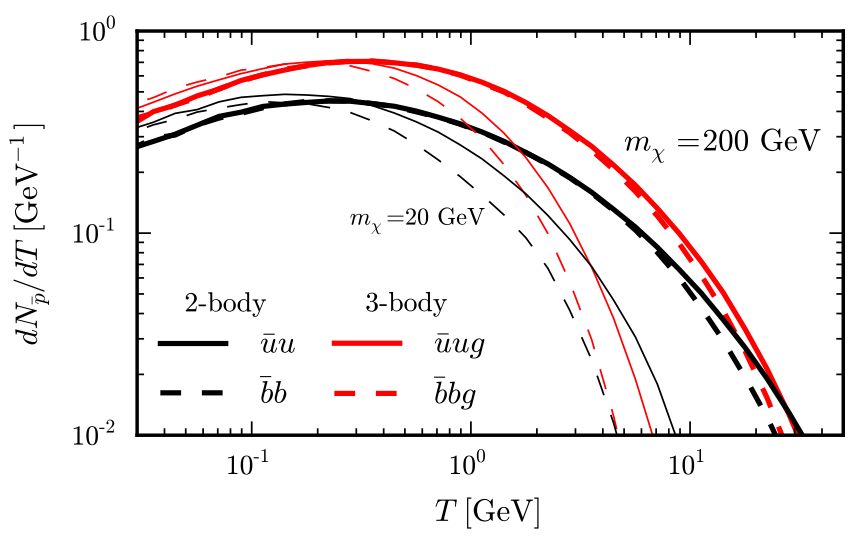

Figure 2: Differential number of antiprotons, per annihilation, from various channels and as function of the antiproton kinetic energy $T$. Red (black) curves show the result from three-body (two-body) final states containing up-quarks (solid lines) and bottom-quarks (dashed lines), respectively. For thick lines, the DM mass is set to $200 \mathrm{GeV}$, for thin lines to $20 \mathrm{GeV}$. We adopted a mass splitting of $\mu=1.2$, but the spectra are practically independent of $\mu$ in the range $\mu=1 \ldots 4$.

\section{Antiproton production and propagation}

The fragmentation of quarks and gluons into color singlets leads to the production of a sizable amount of antiprotons. We derived the antiproton energy spectrum $d N_{\bar{p}} / d T$ (with $T$ denoting kinetic energy) from DM annihilation using Monte Carlo methods. To this end, we simulated the distribution of hard partons from $\chi \chi \rightarrow \bar{q} q g$, following our above analytical results, and used the event generator Pythia 6.4.19 [20] to perform the subsequent parton showering, fragmentation and particle decay.

Our results for the antiproton energy spectra are shown in Fig. 2 for different DM annihilation channels and $\mu=$ 1.2 (assuming that $\mu \equiv \mu_{L} \equiv \mu_{R}$ ); for other values of $\mu$ we obtain very similar results. We find that, in the phenomenologically relevant low energy region, the additional hard gluon in $\bar{q} q g$ final states leads to an enhancement of antiproton production by a factor of up to $\sim 2$ relative to the $\bar{q} q$ final states commonly considered in indirect DM searches. Note that while final state electroweak gauge bosons at first sight can lead to a much larger enhancement of $d N_{\bar{p}} / d T$ for low-energy antiprotons [21], this is only because final state radiation of gluons is already included in the commonly adopted 'two-body' result obtained from Pythia - while that of electroweak gauge bosons is not. Fig. 2 also shows that the amount of antiprotons increases with decreasing quark mass, as is expected for final state radiation (which is dominated by collinear gluons).

Once produced, antiprotons do not travel along straight lines like, e.g., gamma rays, but scatter on randomly distributed galactic magnetic field inhomogeneities. Their propagation can thus nicely be described in terms of a phenomenological diffusion model, the free parameters of

\footnotetext{
${ }^{1}$ Note that there is a typo in Eq. (2) of Ref. [18 - see also Ref. [19.
} 


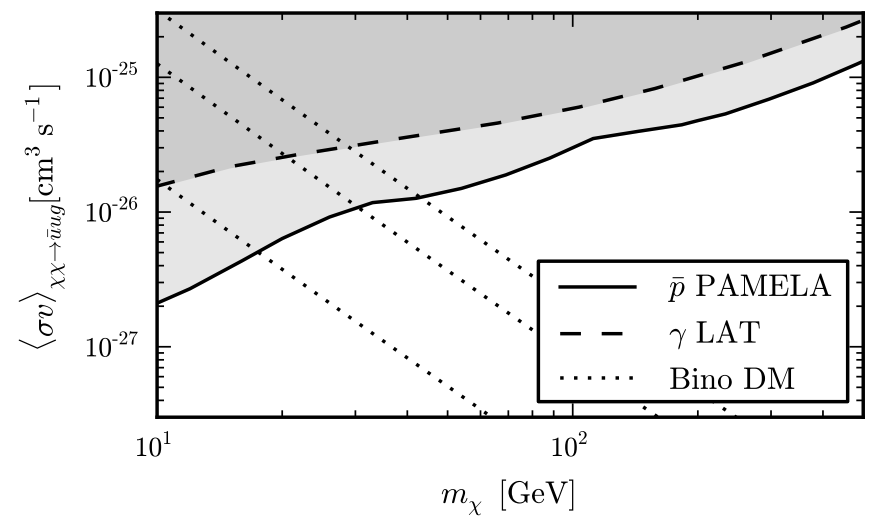

Figure 3: Limits on the DM annihilation cross-section $\langle\sigma v\rangle$, assuming a $100 \%$ branching ratio into $\bar{u} u g$ final states. Gammaray limits (dashed line) are taken from Fermi LAT dwarf galaxy observations [36] and antiproton limits (solid line) are derived from PAMELA data 25]. Dotted lines show the theoretically expected values for Bino DM and mass splittings of $\mu \equiv m_{\tilde{u}}^{2} / m_{\tilde{B}}^{2}=$ $1.01,1.2,2.0$ (from top to bottom); the cosmic-ray limits are practically independent of $\mu$.

which are strongly constrained by other cosmic ray data, in particular the boron over carbon ratio $B / C$ [22]. This allows to predict the expected astrophysical background, consisting of secondary antiprotons mainly produced in the collisions of cosmic ray protons with the interstellar medium, with a remarkable accuracy [23, 24]. The recent cosmic-ray antiproton flux measurements undertaken by the PAMELA experiment over rigidities of $0.35-180$ GV 25] are fully consistent with this background.

The expected flux of primary antiprotons from DM annihilation [26], on the other hand, is subject to greater uncertainties [22]. The reason for this is that the $B / C$ analysis, being restricted to sources in the galactic disk, actually leaves large degeneracies in the diffusion parametersin particular between the size of the diffusion constant and the thickness $L$ of the diffusion zone perpendicular to the galactic plane. While secondary antiprotons are not greatly affected by this degeneracy, DM annihilation happens predominantly in the galactic halo; therefore, a much larger volume of the diffusion zone is probed and the antiproton flux is rather sensitive to the adopted value of $L$. Even though the $B / C$ analysis in principle allows values as small as $L \sim 1 \mathrm{kpc}$, values up to $L \sim 10 \mathrm{kpc}$ are preferred when adding radioactive isotopes to the analysis [27, with similar conclusions following from the consideration of gamma rays [28] and cosmic-ray electrons [29]. Also radio data are in rather strong conflict with a halo size as small as $\sim 1 \mathrm{kpc}$ [30]. In our analysis, we will therefore refer to the 'KRA' model of Ref. 31] and the $B / C$ best fit ('MED') model of Ref. 22, both featuring $L=4 \mathrm{kpc}$, as well as the 'MAX' model of Ref. [22], featuring $L=10 \mathrm{kpc}$.

\section{Indirect detection constraints for $\bar{q} q g$ final states}

We calculated the antiproton flux from DM annihilation, as expected at the top of the atmosphere after propagation, by using DarkSUSY 32 (which implements the procedure described in Refs. [31, 33]). Solar modulation is taken into account by adopting the force-field approximation, with a Fisk potential of $\phi=500 \mathrm{MV}$, but our limits actually do not strongly depend on this choice because they typically do not derive from antiproton energies much smaller then $1 \mathrm{GeV}$. We choose an Einasto profile to model the galactic DM halo, with parameters as used in Ref. [31; for a Navarro-Frenk-White profile, we get very similar results. Furthermore, we assume that the neutralino makes up all of the observed dark matter (noting that, due to the potentially very efficient coannihilation between squarks and neutralinos, this can require a non-thermal production mechanism or a non-standard freeze-out history).

In order to derive limits on the flux of primary antiprotons from DM annihilation we require that the DM signal plus the background of secondary antiprotons does not overshoot the antiproton measurements by PAMELA [25] by more than $3 \sigma$ in any data point; to be conservative, we adopt the minimal astrophysical background flux from Ref. [24]. The resulting limits on the annihilation crosssection $\langle\sigma v\rangle$ are shown in Fig. 3 , as function of the DM mass $m_{\chi}$, for the case of annihilation into $\bar{u} u g$ final states and adopting a mass splitting of $\mu=1.2$; we obtain very similar results for other values of $\mu=1 \ldots 4$ and for the light quark flavors $q=d, s$, but the limits are up to a factor of two weaker in case of the heavier quarks $q=c, b$. We note that these limits are in rather good agreement with previous results, like in Ref. [31, for two-body final states.

Besides antiprotons, the hadronization of quarks and gluons also generates a large amount of gamma rays, which mostly stem from $\pi^{0}$-decay. Using again Pythia, we find reasonable agreement between the gamma-ray energy spectrum from $\bar{q} q g$ and the commonly adopted $\bar{b} b$ spectrum (at the peak of the spectra at $E_{\gamma} \approx m_{\chi} / 20$ the fluxes agree within $20 \%$ for $q=d, u, s, c, b)$. Currently, the Fermi Large Area Telescope (LAT) is measuring the gamma-ray sky with unprecedented precision and strong limits on the DM annihilation cross section were derived from observations of dwarf galaxies [34, 35, 36], galaxy clusters [37, 38] and the isotropic diffuse gamma-ray backround [39]. In Fig. 3 we show the corresponding limits on the annihilation cross-section to $\bar{q} q g$ as derived from the Fermi LAT data by a combined analysis of ten dwarf spheroidal galaxies [36], making use of the above-mentioned similarity between the gamma-ray spectra from $\bar{q} q g$ and $\bar{b} b$ final states. It is reassuring that these limits are almost as strong as our antiproton bounds, since limits derived from antiproton and gamma-ray observations are in principle subject to very different astrophysical uncertainties.

For comparison, we also indicate in Fig. 3 the value of the annihilation cross-section for $\chi \chi \rightarrow \bar{u} u g$ in the case of 


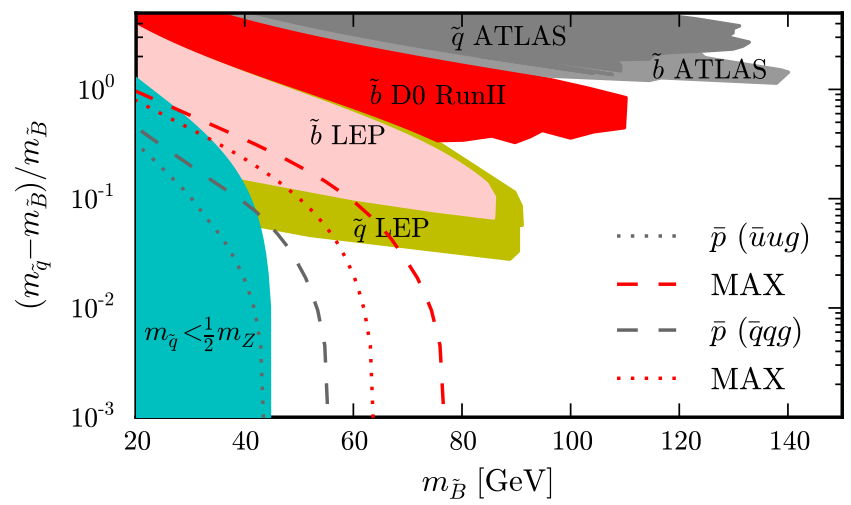

Figure 4: Constraints on squarks degenerate with a Bino LSP, as function of the Bino mass and the mass splitting. The colored areas are excluded by different collider searches as described in the text and the areas below the black lines are constrained by cosmic-ray antiproton observations (for the 'MED' propagation model). Dotted lines correspond to light right-handed up-type squarks, dashed lines assume degeneracy between all $d, u, c$ and $s$-type squarks. The red lines show how the limits strengthen in case of the 'MAX' propagation model.

pure Bino DM, as function of the neutralino mass $m_{\chi}$ and the mass splittings $\mu$. Depending on the value of $\mu$, we find that DM masses up to $\sim 50 \mathrm{GeV}$ can be excluded by antiproton and gamma-ray limits; this will be confronted with collider limits in the next section.

\section{Collider constraints on new colored particles}

In general it is difficult to directly constrain the DM mass with collider experiments. On the other hand, light charged and colored particles can be searched for with many experiments, and in the recent past the LHC has put strong lower limits on squark masses, reaching up to $\sim 900$ $\mathrm{GeV}$ [3. However, these constraints are highly model dependent and, in the case of supersymmetry, depend crucially on the supersymmetric particle mass spectrum: very heavy gluinos, e.g., would suppress the squark production in hadron colliders. Additionally, in the parameter region in which the mass difference between squarks and the lightest superpartner (which is the DM candidate) is very small, the constraints from collider experiments are evaded since the energy of QCD jets and missing transverse momentum that stem from $\tilde{q} \rightarrow q \chi$ are small, and these events therefore cannot pass triggers and cuts. In general, for light squarks with masses less than $\sim 100 \mathrm{GeV}$, the constrains are very severe because such light particles are produced at LEP experiments. Even if the mass splitting is relatively small, $\sim 5-10 \mathrm{GeV}$, a large parameter region of the squark mass in which it can be pair produced is constrained [9, 10, 11, 12, 13]. For squarks that are lighter than $\sim m_{Z} / 2$, the $Z$ boson decay width generally gives the strongest constraints 40.

Light squarks are also indirectly constrained by experiments due to the contribution of loop diagrams; con- tributions of these particles have e.g. a large effect on electroweak precision measurements and flavor changing neutral currents 41]. However, since these limits on the squark mass are generically very model-dependent, we will not discuss them in this Letter.

In Fig. 4 we show the constraints on squarks degenerate with a Bino LSP that derive from collider null searches, compared with the constraints from cosmic-ray antiproton observations. In particular, we show the following limits: squark and sbottom mass constraints from the LEP experiments [11, 13, sbottom mass constraints from the Tevatron D0 experiment [42] and constraints from the LHC ATLAS experiment on squark and sbottom masses [5, 43. (for constraints from the CMS experiment, see also [44]). Concerning the antiproton constraints, we consider two limiting cases: (1) only the right-handed up-type squark is light (dotted lines), (2) $d, u, c$ and $s$-type squarks are mass degenerate and light (dashed lines). The areas below the corresponding curves are excluded by antiproton measurements with PAMELA, where we adopted the 'MED' propagation model (for the black curves).

As expected, these limits are strongest for small mass splittings and are hence constraining the parameter space from a direction that is complementary to the collider limits. As can be seen from Fig. 4, in case of light up-type squarks and small mass-splitting, we can exclude Bino masses up to $45 \mathrm{GeV}$ (the 'KRA' propagation model gives limits that are about $10 \mathrm{GeV}$ stronger); under favorable astrophysical conditions (the 'MAX' model) these limits could strengthen to above $60 \mathrm{GeV}$. If all $d, u, c$ and $s$-type squarks are mass degenerate, the lower limits on the Bino mass increase to around 55-80 GeV, depending on the astrophysical scenario. Hence, we find that cosmic-ray observations, in particular the observations of antiprotons, can be a powerful probe of a parameter region of DM models that is generically difficult to access directly with colliders.

\section{Discussion}

While we have demonstrated that favorable astrophysical conditions, in particular a large diffusive halo like in the 'MAX' model, could significantly improve the constraining power of indirect DM searches with antiprotons, we would like to stress that prospects could be even better when taking into account the possibility that the DM distribution does not follow a smooth Einasto profile. For an adiabatically compressed profile as in Refs. [45, 46, which has been argued to result from the gravitational impact of the observed galactic distribution of baryons on the DM profile, e.g., we find a further enhancement of the antiproton flux by a factor of about $4-5$ at $1 \mathrm{GeV}$, which corresponds to an improvement for the lower bounds on the DM mass by about $20-30 \mathrm{GeV}$. Another rather large enhancement could result from the fact that DM is not expected to be distributed smoothly, but to cluster in the form of small subhalos [4]. The resulting effective 'boost-factor' of the antiproton flux could in principle be quite large [24, 48, 49], 
though one should note that statistically a much smaller value (corresponding to a factor of a few enhancement) is expected when extrapolating the results from $N$-body simulations of gravitational clustering [50. Here, we will not study such effects in more detail but simply note that already a moderate boost-factor of 5 (10) would improve the lower limit on $m_{\chi}$ by roughly 25 (40) GeV.

Future prospects for indirect DM searches with antiprotons may be even more promising, given that new data with excellent statistics can soon be expected from the recently launched AMS-02 experiment [51. This can only lead to better limits on exotic contributions to the cosmic-ray antiproton flux, however, if complemented with an improved understanding of the astrophysical background. It was estimated that this could strengthen current bounds by about one order of magnitude [31], which would make it possible to probe Bino masses up to $100 \mathrm{GeV}$ and beyond, depending on the size of the mass-splitting, the astrophysical propagation model and the nature of the light squark flavors. In addition, as can be seen from Fig. 3 , gamma-ray observations are also quite constraining for the channels considered here; the current limits from Fermi LAT observations will strengthen further with a better understanding of the Galactic center region and more accumulated data.

In this Letter we have concentrated on Bino-like neutralinos, which are probably the most difficult to probe due to their relatively weak interactions with fermions and their suppressed annihilation signal in cosmic rays. Let us stress that the discussed channel $\bar{q} q g$ can also be relevant for Wino- or Higgsino-like neutralinos, in which case it is however often dominated by other two-body channels like, in particular, $\chi \chi \rightarrow Z Z$ and $W^{+} W^{-}$. In these cases, masses below a few hundred $\mathrm{GeV}$ are difficult to reconcile with existing gamma-ray or antiproton limits, see e.g. Refs. 31, 36, and we leave a phenomenological exploration of $\bar{q} q g$ final states at $\mathcal{O}(100 \mathrm{GeV}-1 \mathrm{TeV})$ masses for future work.

Finally, let us mention another important possibility to search for degenerate mass spectra. The squark-neutralino degeneracy is also expected to yield strong constraints from direct dark matter searches because the neutralinonucleus scattering cross section can be resonantly enhanced due to the $s$-channel squark exchange diagram. In fact, the XENON100 experiment can have sufficient sensitivity for DM masses around $50 \mathrm{GeV}$ [52]. A detailed analysis of direct detection constraints for any possible squarkneutralino degeneracy, however, is beyond the scope of this letter and left for future work (but see Ref. [53]).

\section{Conclusions}

In this Letter, we have described a DM annihilation channel, and discussed its phenomenological implications in some detail, that so far has hardly been explored in the context of indirect dark matter searches. If DM is a Majorana fermion $\chi$, or a scalar, direct annihilation via $\chi \chi \rightarrow \bar{q} q$ is strongly helicity suppressed. In this case, the overall annihilation rate today can be dominated by internal gluon Bremsstrahlung, $\chi \chi \rightarrow \bar{q} q g$, and can be boosted by several orders of magnitude with respect to the treelevel result; while this enhancement is much smaller in the early universe, during freeze-out, it can also have a nonnegligible effect on the relic density [16, 17, 54]. The precise branching ratios depend on the difference between the DM particle's mass $m_{\chi}$ and the squark mass $m_{\tilde{q}}$; the process $\chi \chi \rightarrow \bar{q} q g$ becomes strongest in the mass degenerate case, i.e. for $m_{\tilde{q}} / m_{\chi} \simeq 1$.

We studied the spectra of cosmic rays generated by this channel, and found that the energy spectrum of antiprotons coming from $\bar{q} q g$ is generically enhanced with respect to the spectrum from $\bar{q} q$ final states (Fig. 2). The corresponding limits on the annihilation cross-section for $\chi \chi \rightarrow \bar{q} q g$ are hence somewhat stronger than limits on the traditional $\bar{b} b$ or $W^{+} W^{-}$final states (Fig. 3). From this figure we can see, as noted before [31, 55, 56, 57, that antiprotons are most constraining for light DM masses, which in particular seems to disfavor the simplest DM interpretations of recent results from direct DM detection experiments [58, 59, 60].

Even more interestingly, the region of small mass splittings between squarks and the LSP (where the annihilation fluxes are largest) is precisely the region that is generically difficult to probe directly with collider searches for squarks; this is because the energy stored in hadronic jets coming from the decay $\tilde{q} \rightarrow \chi q$ becomes too small to pass the trigger selection in this limit. In this sense, indirect DM searches are complementary to collider searches and constrain a part of the parameter space that is not directly accessible by either LEP, Tevatron or LHC (Fig. 4) ${ }^{2}$ Depending on the cosmic-ray propagation model, these limits can exclude Bino masses up to $45-80 \mathrm{GeV}$ in the degenerate case. Ongoing AMS-02 measurements are expected to strengthen the limits on the annihilation cross-section by up to an order of magnitude (see e.g. Ref. [31]), which will make it possible to probe Bino masses of $100 \mathrm{GeV}$ and beyond.

Note added. During the final stages of this work, we became aware of another project analyzing the annihilation of DM into $\bar{q} q g$ final states 61 .

Acknowledgments. We would like to thank Piero Ullio for providing the antiproton propagation routines that were used in Ref. [31, which will eventually become publicly available with the next release of DarkSUSY 32. We also would like to thank Shoji Asai, Keisuke Fujii and Yasuhiro Shimizu for useful comments. M.A. and T.B. acknowledge

\footnotetext{
${ }^{2}$ Note that also in the situation where the scalar tops are heavy, which generally helps to lift the lightest neutral Higgs boson mass to the high value of $\sim 125 \mathrm{GeV}$ consistent with recent indications found by both ATLAS and CMS, our results from indirect DM searches would not change and constitute important independent limits on light first or second generation squarks.
} 
support from the German Research Foundation (DFG) through Emmy Noether grant BR 3954/1-1.

\section{References}

[1] G. Aad et al. [Atlas Collaboration], JHEP 1111, 099 (2011) arXiv:1110.2299 [hep-ex]].

[2] S. Chatrchyan et al. [CMS Collaboration], Phys. Rev. Lett. 107, 221804 (2011) arXiv:1109.2352 [hep-ex]].

[3] G. Aad et al. [ATLAS Collaboration], arXiv:1109.6572 [hep-ex].

[4] CMS Collaboration, CMS PAS SUS-11-009 (2011).

[5] G. Aad et al. [ATLAS Collaboration], ATLAS-CONF-2011-155 (2011).

[6] S. Chatrchyan et al. [CMS Collaboration], Phys. Rev. D 85, 012004 (2012) arXiv:1107.1279 [hep-ex]].

[7] T. J. LeCompte and S. P. Martin, Phys. Rev. D 84 (2011) 015004 arXiv:1105.4304 [hep-ph]].

[8] T. J. LeCompte and S. P. Martin, arXiv:1111.6897 [hep-ph].

[9] A. Heister et al. [ALEPH Collaboration], Phys. Lett. B 537 (2002) 5 hep-ex/0204036.

[10] G. Abbiendi et al. [OPAL Collaboration], Phys. Lett. B $\mathbf{5 4 5}$ (2002) 272 [Erratum-ibid. B 548 (2002) 258] hep-ex/0209026].

[11] P. Achard et al. [L3 Collaboration], Phys. Lett. B 580 (2004) 37 hep-ex/0310007.

[12] J. Abdallah et al. [DELPHI Collaboration], Eur. Phys. J. C 31 (2003) 421 hep-ex/0311019.

[13] LEPSUSYWG, ALEPH, DELPHI, L3 and OPAL experiments, note LEPSUSYWG/04-02.1.

[14] G. Bertone, D. Hooper, J. Silk, Phys. Rept. 405 (2005) 279 hep-ph/0404175.

[15] L. Bergström, Phys. Lett. B 225, 372 (1989).

[16] R. Flores, K. A. Olive and S. Rudaz, Phys. Lett. B 232, 377 (1989).

[17] M. Drees, G. Jungman, M. Kamionkowski and M. M. Nojiri, Phys. Rev. D 49, 636 (1994) hep-ph/9306325.

[18] L. Bergström, T. Bringmann and J. Edsjö, Phys. Rev. D 78, 103520 (2008) arXiv:0808.3725 [astro-ph]].

[19] N. F. Bell, J. B. Dent, A. J. Galea, T. D. Jacques, L. M. Krauss and T. J. Weiler, Phys. Lett. B 706 (2011) 6 arXiv:1104.3823 [hep-ph]].

[20] T. Sjöstrand, S. Mrenna and P. Z. Skands, JHEP 0605 (2006) 026 hep-ph/0603175.

[21] P. Ciafaloni, D. Comelli, A. Riotto, F. Sala, A. Strumia and A. Urbano, JCAP 1103, 019 (2011) arXiv:1009.0224 [hep-ph]].

[22] F. Donato, N. Fornengo, D. Maurin and P. Salati, Phys. Rev. D 69, 063501 (2004) astro-ph/0306207.

[23] F. Donato, D. Maurin, P. Salati, A. Barrau, G. Boudoul, R. Taillet, Astrophys. J. 563, 172 (2001) astro-ph/0103150.

[24] T. Bringmann and P. Salati, Phys. Rev. D 75, 083006 (2007) astro-ph/0612514.

[25] O. Adriani et al. [PAMELA Collaboration], Phys. Rev. Lett. 105 (2010) 121101 arXiv:1007.0821 [astro-ph.HE]].

[26] J. Silk and M. Srednicki, Phys. Rev. Lett. 53, 624 (1984).

[27] A. Putze, L. Derome and D. Maurin, Astron. Astrophys. 516, A66 (2010) arXiv:1001.0551 [astro-ph.HE]].

[28] D. Timur, F. Armand, M. Pohl and P. Salati, Astron. Astrophys. 531, A37 (2011) arXiv:1102.0744 [astro-ph.HE]].

[29] T. Delahaye, F. Donato, N. Fornengo, J. Lavalle, R. Lineros, P. Salati and R. Taillet, Astron. Astrophys. 501, 821 (2009) arXiv:0809.5268 [astro-ph]]; J. Lavalle, Mon. Not. Roy. Astron. Soc. 414, 985L (2011) arXiv:1011.3063 [astro-ph.HE]].

[30] T. Bringmann, F. Donato and R. A. Lineros, JCAP 1201, 049 (2012) arXiv:1106.4821 [astro-ph.GA]].

[31] C. Evoli, I. Cholis, D. Grasso, L. Maccione and P. Ullio, arXiv:1108.0664 [astro-ph.HE].

[32] P. Gondolo, J. Edsjö, P. Ullio, L. Bergström, M. Schelke and E. A. Baltz, JCAP 0407, 008 (2004) astro-ph/0406204; P. Gondolo, J. Edsjö, L. Bergström, P. Ullio, M. Schelke, E. A. Baltz, T. Bringmann and G. Duda, http://www.physto.se/ edsjo/darksusy
[33] L. Bergström, J. Edsjö and P. Ullio, Astrophys. J. 526 (1999) 215 astro-ph/9902012.

[34] A. A. Abdo, M. Ackermann, M. Ajello, W. B. Atwood, L. Baldini, J. Ballet, G. Barbiellini and D. Bastieri et al., Astrophys. J. 712 (2010) 147 arXiv:1001.4531 [astro-ph.CO]].

[35] A. Geringer-Sameth and S. M. Koushiappas, Phys. Rev. Lett. 107 (2011) 241303 arXiv:1108.2914 [astro-ph.CO]].

[36] M. Ackermann et al. [Fermi-LAT Collaboration], Phys. Rev. Lett. 107, 241302 (2011) arXiv:1108.3546 [astro-ph.HE]].

[37] M. Ackermann, M. Ajello, A. Allafort, L. Baldini, J. Ballet, G. Barbiellini, D. Bastieri and K. Bechtol et al., JCAP 1005 (2010) 025 arXiv:1002.2239 [astro-ph.CO]].

[38] X. Huang, G. Vertongen and C. Weniger, JCAP 1201 (2012) 042 arXiv:1110.1529 [hep-ph]].

[39] A. A. Abdo et al. [Fermi-LAT Collaboration], JCAP 1004 (2010) 014 arXiv:1002.4415 [astro-ph.CO]].

[40] K. Nakamura et al. [Particle Data Group], J. Phys. G 37 (2010) 075021.

[41] M. J. Ramsey-Musolf and S. Su, Phys. Rept. 456, 1 (2008) hep-ph/0612057.

[42] V. M. Abazov et al. [D0 Collaboration], Phys. Lett. B 693 (2010) 95 arXiv:1005.2222 [hep-ex]].

[43] G. Aad et al. [ATLAS Collaboration], arXiv:1112.3832 [hep-ex].

[44] CMS Collaboration, CMS-PAS-SUS-11-004 (2011).

[45] O. Y. Gnedin and J. R. Primack, Phys. Rev. Lett. 93, 061302 (2004) astro-ph/0308385.

[46] M. Gustafsson, M. Fairbairn and J. Sommer-Larsen, Phys. Rev. D 74, 123522 (2006) astro-ph/0608634.

[47] L. Bergström, J. Edsjö, P. Gondolo and P. Ullio, Phys. Rev. D 59, 043506 (1999) astro-ph/9806072.

[48] P. Brun, G. Bertone, J. Lavalle, P. Salati and R. Taillet, Phys. Rev. D 76, 083506 (2007) arXiv:0704.2543 [astro-ph]].

[49] M. Regis and P. Ullio, arXiv:0907.5093 [astro-ph.GA].

[50] L. Pieri, J. Lavalle, G. Bertone and E. Branchini, Phys. Rev. D 83, 023518 (2011) arXiv:0908.0195 [astro-ph.HE]].

[51] http://www.ams02.org/

[52] E. Aprile et al. [XENON100 Collaboration], Phys. Rev. Lett. 107 (2011) 131302 arXiv:1104.2549 [astro-ph.CO]].

[53] J. Hisano, K. Ishiwata and N. Nagata, Phys. Lett. B 706, 208 (2011) arXiv:1110.3719 [hep-ph]].

[54] V. Barger, W. -Y. Keung, H. E. Logan and G. Shaughnessy, Phys. Rev. D 74, 075005 (2006) hep-ph/0608215.

[55] A. Bottino, F. Donato, N. Fornengo and P. Salati, Phys. Rev. D 72, 083518 (2005) hep-ph/0507086.

[56] T. Bringmann, arXiv:0911.1124 [hep-ph].

[57] J. Lavalle, Phys. Rev. D 82, 081302 (2010) arXiv:1007.5253 [astro-ph.HE]].

[58] R. Bernabei, P. Belli, F. Cappella, R. Cerulli, C. J. Dai, A. d'Angelo, H. L. He and A. Incicchitti et al., Eur. Phys. J. C 67, 39 (2010) arXiv:1002.1028 [astro-ph.GA]].

[59] C. E. Aalseth, P. S. Barbeau, J. Colaresi, J. I. Collar, J. Diaz Leon, J. E. Fast, N. Fields and T. W. Hossbach et al., Phys. Rev. Lett. 107 (2011) 141301 arXiv:1106.0650 [astro-ph.CO]].

[60] G. Angloher, M. Bauer, I. Bavykina, A. Bento, C. Bucci, C. Ciemniak, G. Deuter and F. von Feilitzsch et al., arXiv:1109.0702 [astro-ph.CO].

[61] M. Garny, A. Ibarra and S. Vogl, arXiv:1112.5155 [hep-ph]. 